\title{
NOUVELLE
}

\section{Les tumeurs gliales diffuses de l'adulte}

\section{Hétérogénéité génétique et implications thérapeutiques}

Maïté Verreault ${ }^{1,2,3}$, Jean-Yves Delattre ${ }^{1,2,3,4}$, Ahmed Idbaih ${ }^{1,2,3,4}$
${ }^{1}$ Université Pierre et Marie Curie Paris-6,

Centre de Recherche de l'Institut du cerveau et de la moelle épinière (CRICM), UMRS 975, 47-83, boulevard de l'Hôpital, 75013 Paris, France.

${ }^{2}$ Inserm U975, 47-83, boulevard de I'Hôpital, 75013 Paris, France. ${ }^{3}$ CNRS, UMR 7225, 47, boulevard de l'Hôpital, 75013 Paris, France. ${ }^{4}$ AP-HP, Groupe hospitalier Pitié-Salpêtrière, Service de neurologie Mazarin, 47-83, boulevard de l'Hôpital, 75013 Paris, France.

maiteverreault@gmail.com
$>$ Les gliomes ou tumeurs gliales sont la forme la plus fréquente de tumeur cérébrale primitive (c'est-à-dire non métastatique) chez l'adulte. Aux États-Unis et en Europe, l'incidence de ce type de cancer est de l'ordre de 2,5 nouveaux cas pour 100000 personnes par année [1]. Certaines prédispositions génétiques héréditaires aux gliomes ont été identifiées. Néanmoins, la majorité des gliomes (>90\%) apparaissent de manière sporadique, en dehors de ces contextes génétiques, suggérant une combinaison de facteurs de risque intrinsèques (prédisposition ou susceptibilité génétique) et extrinsèques (facteurs environnementaux) dans le développement de ces tumeurs. Au cours des dernières décen- nies, la recherche a permis des avancées majeures dans la connaissance de la biologie des gliomes, laissant présager le développement de nouveaux outils diagnostiques et de nouveaux traitements plus efficaces et moins toxiques pour les patients. Parallèlement, ces découvertes ont également révélé la complexité et I'hétérogénéité des tumeurs gliales sur le plan moléculaire et cellulaire.

\section{Classification des tumeurs gliales diffuses et hétérogénéité intertumorale}

La classification de l'Organisation mondiale de la santé (OMS) distingue sept principaux types de tumeurs gliales diffuses en fonction du type de cellules tumorales et du grade

\begin{tabular}{|c|c|c|c|}
\hline Phénotype & Grade & $\begin{array}{l}\text { Sous-types } \\
\text { de gliomes diffus }\end{array}$ & $\begin{array}{l}\text { Biomarqueurs } \\
\text { pronostiques et/ou } \\
\text { prédictifs favorables }\end{array}$ \\
\hline \multirow[t]{8}{*}{ Astrocytome } & II & Astrocytome de bas grade & \multirow{3}{*}{$\begin{array}{l}\text { IDH1/IDH2 muté } \\
\text { Promoteur de MGMT méthylé }\end{array}$} \\
\hline & & & \\
\hline & III & Astrocytome anaplasique & \\
\hline & \multirow[t]{5}{*}{ IV } & \multirow[t]{5}{*}{ Glioblastome } & Primaire : \\
\hline & & & Promoteur de MGMT méthylé \\
\hline & & & Secondaire: \\
\hline & & & IDH1/IDH2 muté \\
\hline & & & Promoteur de MGMT méthylé \\
\hline \multirow[t]{3}{*}{ Oligodendrogliome } & II & Oligodendrogliome de bas grade & \multirow{2}{*}{$\begin{array}{l}\text { IDH1/IDH2 muté } \\
\text { lp/19q codélété }\end{array}$} \\
\hline & & & \\
\hline & III & Oligodendrogliome anaplasique & Promoteur de MGMT méthylé \\
\hline \multirow[t]{3}{*}{ Oligoastrocytome } & II & Oligoastrocytome de bas grade & \multirow{2}{*}{$\begin{array}{l}\text { IDH1/IDH2 muté } \\
\text { lp/19q codélété }\end{array}$} \\
\hline & & & \\
\hline & III & Oligoastrocytome anaplasique & Promoteur de MGMT méthylé \\
\hline
\end{tabular}

Tableau I. Les sept grands sous-types de gliomes diffus et classification histomoléculaire associée à ces sous-types. Les informations présentées sont extraites de [14-18]. de malignité. Trois types de gliomes sont individualisés en fonction des similitudes morphologiques entre les cellules gliales normales et les cellules gliales tumorales: (1) les astrocytomes, (2) les oligodendrogliomes et (3) les oligoastrocytomes (ou gliomes mixtes). Dans les gliomes diffus, I'OMS distingue trois grades de malignité: (1) le grade II (gliomes diffus de bas grade), (2) le grade III (gliomes anaplasiques) et (3) le grade IV (glioblastomes) (Tableau I). Le grade de malignité est déterminé par le degré de différenciation de la tumeur et sa cellularité, ainsi que la présence d'atypies cytonucléaires, d'activité mitotique, de prolifération microvasculaire et de nécrose.

Bien que constituant l'étalon or pour le diagnostic des tumeurs gliales, la classification de l'OMS est basée sur des critères subjectifs et peu reproductibles dans certains sous-types tumoraux [2]. Elle demeure également imparfaite pour prédire le pronostic et la réponse individuelle aux traitements. En effet, au sein d'un même sous-type histologique, les tumeurs ont des évolutions très différentes.

D'importants efforts de recherche ont donc été menés au cours des dernières années afin d'identifier des biomarqueurs moléculaires (anomalie génétique, anomalie de I'ARN et/ou anomalie protéique) informatifs au niveau diagnostique, pronostique, prédictif ou de susceptibilité/prédisposition permettant d'identifier des sous-groupes histomoléculaires plus homogènes. La classification histomoléculaire des tumeurs (Tableau I) s'est avérée une approche intéressante pour affiner le diagnostic, et pour prédire le pronostic et la réponse 
aux traitements antitumoraux en cancérologie générale et en neuro-oncologie. La codélétion des bras chromosomiques $1 p$ et $19 q$ est présente dans la majorité des oligodendrogliomes et associée à un meilleur pronostic et à une meilleure réponse à la chimiothérapie cytotoxique [3]. En effet, des données récentes indiquent que les tumeurs oligodendrogliales anaplasiques présentant la codélétion $1 p / 19 q$ bénéficient clairement d'une chimiothérapie par PCV (combinaison de procarbazine, vincristine et (CNU), néoadjuvante ou adjuvante à la radiothérapie encéphalique. En revanche, ce bénéfice n'est pas démontré dans les tumeurs morphologiquement identiques ne présentant pas la codélétion $1 p / 19 q[4]$. De même, les mutations des gènes IDHI ou IDH2 induisant entre autres la production de l'oncométabolite 2-hydroxyglutarate [5], ou la méthylation du promoteur du gène MGMT empêchant l'expression de l'enzyme de réparation de I'ADN MGMT [6, 7], sont prédictifs d'une survie plus longue et/ou d'une meilleure réponse à la chimiothérapie alkylante comme le témozolomide dans différents sous-types de gliomes. $\varepsilon$ n raison de son utilité, la classification histomoléculaire des gliomes est appelée à se développer de plus en plus.

\section{Hétérogénéité intratumorale}

En plus de l'hétérogénéité intertumorale, les gliomes présentent également une hétérogénéité intratumorale qui a suscité beaucoup de travaux de recherche ces dernières années. En effet, au sein d'une même tumeur gliale, il existe plusieurs populations ou sous-clones de cellules tumorales aux caractéristiques cellulaires et moléculaires hétérogènes. Cette hétérogénéité moléculaire et cellulaire permettrait à la tumeur de se développer dans différents environnements ou dans un environnement dynamique (face à I'hypoxie, au stress métabolique, à la chimiothérapie, à la radiothérapie ou à une thérapie ciblée contre un gène).

Plusieurs populations cellulaires distinctes coexistent selon la région de la tumeur: le corps proliférant, le front invasif, la niche hypoxique, les cellules initiatrices de gliomes (CIG) ou la population de cellules endothéliales formant la microvasculature tumorale (Figure IA). À cette hétérogénéité cellulaire s'ajoute une hétérogénéité moléculaire au sein d'une même région tumorale, entre différentes cellules voisines. Ainsi, l'amplification de multiples oncogènes (EGFR, MET ou PDGFR $\alpha$ [voir Glossaire]) a été observée simultanément et de façon mutuellement exclusive dans des cellules gliomateuses voisines [8] (Figure 1A). La méthylation du promoteur de MGMT est également variable d'une cellule tumorale à l'autre [9]. Ces observations suggèrent des mécanismes moléculaires et cellulaires d'initiation et de progression tumorales complexes, ainsi qu'une coopération des différents sous-clones tumoraux entre eux et avec leur environnement afin de pérenniser la tumeur [10].

\section{Implications de l'hétérogénéité des tumeurs gliales}

Implications cliniques de l'hétérogénéité intertumorale au sein d'un sous-groupe histologique

Le diagnostic clinique, radiologique et histologique des tumeurs gliales est insuffisant pour une prise en charge optimale des patients, notamment pour prédire le pronostic et guider les traitements. Les données histomoléculaires permettant d'identifier des sous-groupes tumoraux plus homogènes deviennent de plus en plus cruciales pour compléter le diagnostic clinico-radio-histologique. Ainsi, les patients présentant une tumeur oligodendrogliale anaplasique avec la codélétion $1 p / 19 q$ sont dorénavant traités par une chirurgie, une radiothérapie encéphalique et une chimiothérapie dont le bénéfice dans ce sous-groupe histomoléculaire a récemment été démontré de manière formelle. Cependant, certains biomarqueurs prédictifs ne permettent pas encore de moduler le traitement. Ainsi, en l'absence d'alternative thérapeutique, les patients atteints de gliomes présentant un promoteur non méthylé du gène MGMT sont actuellement pris en charge de la même façon que ceux atteints d'une tumeur présentant un promoteur méthylé, ceci à l'exception des patients inclus dans des essais cliniques. $\varepsilon n$ théorie, les thérapies moléculaires ciblées apparaissent extrêmement prometteuses si elles sont délivrées aux tumeurs candidates porteuses de l'altération moléculaire cible. Pourtant, les thérapies moléculaires ciblées telles que l'erlotinib (Tarceva ${ }^{\circledR}$, Roche) dirigé contre l'EGFR et l'imatinib mesylate (Gleevec ${ }^{\circledR}$, Novartis) dirigé contre le PDGFR $\alpha$ et c-Kit, des protéines fréquemment activées dans les glioblastomes, ont démontré une efficacité thérapeutique modeste chez un nombre limité de patients présentant un gliome. De plus, les facteurs moléculaires prédictifs d'une réponse à ces thérapies innovantes n'ont pas pu être identifiés de manière solide, et ne semblaient pas seulement être associés à la présence de l'altération cible théorique [11, 12]. L'absence d'effet thérapeutique clair dans ces études pourrait donc s'expliquer en partie par l'hétérogénéité moléculaire des tumeurs incluses dans les essais cliniques. Ces exemples démontrent l'importance de l'identification de facteurs moléculaires prédictifs afin de mieux définir les patients susceptibles de bénéficier de ces traitements.

\section{Implications cliniques de l'hétérogénéité intratumorale des tumeurs gliales}

L'analyse histomoléculaire d'une tumeur basée sur une biopsie provenant d'une seule région tumorale comporte nécessairement un biais spatial et ne reflète que partiellement la tumeur. Cette considération est d'autant plus importante à prendre en compte que le choix du traitement est basé sur cette analyse anatomopathologique. Bien que difficiles à réaliser en pratique, de multiples biopsies pourraient être nécessaires pour avoir un portrait représentatif de la tumeur dans sa complexité. L'étude des biomarqueurs dans des cellules individuelles par des techniques comme le FISH (fluorescent in situ hybridization) ou l'immunohistochimie pourraient apporter des informations 


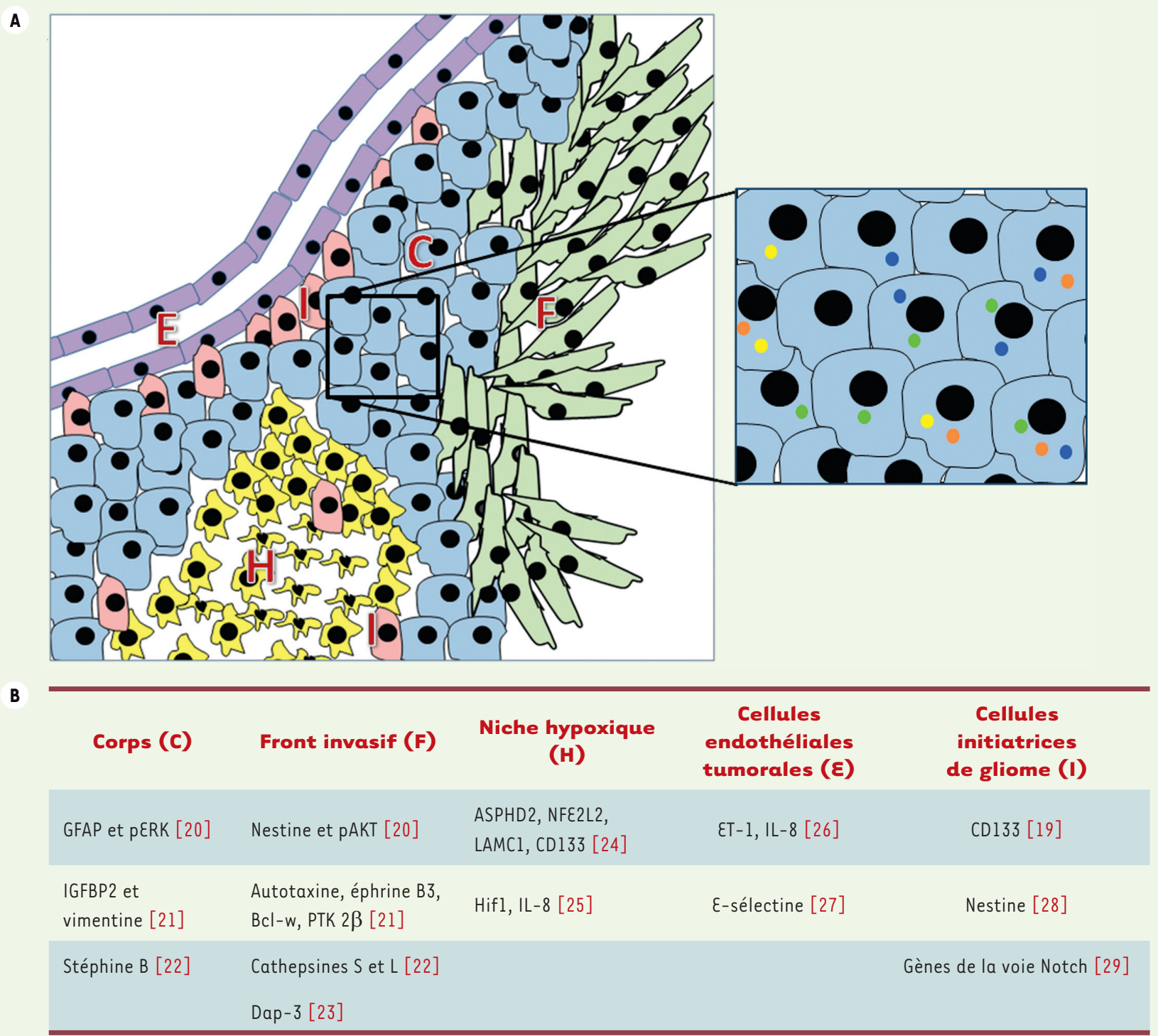

Figure 1. L'hétérogénéité des tumeurs gliales. A. Dans cet exemple, un glioblastome est représenté avec le corps de la tumeur (C), le front invasif $(F)$, une niche hypoxique $(H)$, les cellules endothéliales $(\varepsilon)$ formant la microvasculature tumorale, ainsi que des cellules initiatrices de gliomes (I) se trouvant typiquement dans les niches périvasculaires ou hypoxiques. Les sous-populations tumorales présentent un aspect morphologique différent. Encadré. À l'intérieur même d'une sous-population tumorale, les cellules présentent de façon hétérogène des altérations moléculaires, telles que l'amplification de l'EGFR (points oranges), de la MET (points jaunes), du PDGFR $\alpha$ (points verts) ou la méthylation du promoteur de MGMT (points bleus). B. Les différentes sous-populations tumorales expriment des gènes différents en faveur d'un comportement biologique différent [19-29] (voir Glossaire pour définition des abréviations).

plus précises sur les différentes composantes cellulaires de la tumeur. Enfin, l'imagerie moléculaire permettrait également la détection de manière non invasive des différentes altérations moléculaires au sein d'une tumeur gliale.

Cette hétérogénéité tumorale constitue un défi thérapeutique. En effet, un important effort de recherche est réalisé pour identi- fier les cibles thérapeutiques moléculaires et cellulaires clés dans la masse tumorale hétérogène (Figure IB), c'est-à-dire les anomalies dites pilotes (driver) en opposition à celles dites annexes (passenger). Ces anomalies moléculaires pilotes seraient à viser en priorité.

Une combinaison de plusieurs agents thérapeutiques, des agents multicibles, et/ou des agents inhibant des acteurs très distaux des cascades de signalisation intracellulaires (mTOR, NFKB) pourraient également s'avérer plus efficace que l'utilisation d'un seul agent. En effet, ces approches semblent être une piste intéressante pour répondre à et anticiper les multiples stratégies de résistance aux traitements qui seront développées par la tumeur. 


\section{GLOSSAIRE}

$1 p / 19 q$ : codélétion des bras chromo-

somiques $1 p$ et $19 q$

ASPHD2 : aspartate beta-hydroxylase

domain containing 2

Bcl-w : B cell lymphoma w

CIG : cellules initiatrices de gliomes

Dap-3 : death-associated protein 3

EGFR : epidermal growth factor receptor

हT-1: endothelin 1

GFAP : glial fibrillary acidic protein

HIF1 : hypoxia-inducible factor 1

IDH1/2 : isocitrate dehydrogenase 1/2

IGFBP2 : insulin-like growth factor

binding protein 2

IL-8 : interleukin 8

LAMC1 : laminin gamma 1

MGMT : O(6)-methylguanine-DNA-

methyltransferase

mTOR : mammalian target of rapamycin

NFE2L2 : nuclear factor (erythroid-

derived 2)-like 2

NFKB : nuclear factor $K B$

OMS : Organisation mondiale de la santé

PAKT : forme phosphorylée de AKT

PERK : forme phosphorylée de extracel-

lular signal-regulated kinase

PDGFR $\alpha$ : platelet-derived growth fac-

tor receptor $\alpha$

PTK2 $\boldsymbol{\beta}$ : protein tyrosine kinase $2 \beta$

Plusieurs auteurs ont suggéré qu'une thérapie ciblant les cellules initiatrices de gliomes (CIG) pourrait être plus efficace qu'une thérapie affectant le corps de la tumeur qui comporte des cellules tumorales plus différenciées. En effet, les CIG seraient responsables de l'initiation tumorale, de la résistance aux traitements et de la récidive tumorale, quoique cette idée soit encore discutée [13]. Bien que ces cellules soient bien définies sur le plan fonctionnel (capacité d'autorenouvellement, de différenciation et de tumorigénicité), la question des marqueurs spécifiques permettant de les identifier reste débattue et de nombreuses équipes de recherche dans le monde travaillent à mieux caractériser cette souspopulation. Finalement, les interactions entre les différentes sous-populations de cellules formant les gliomes ne sont pas complètement élucidées et demeurent un sujet de recherche prioritaire.
En conclusion, les tumeurs gliales présentent une hétérogénéité intertumorale au sein d'un même sous-groupe histologique, et intratumorale au sein d'une même tumeur gliale. Les données biologiques permettent de disséquer cette hétérogénéité intertumorale afin d'établir des groupes de tumeurs homogènes sur le plan clinique, radiologique, histologique, cellulaire et moléculaire pour une meilleure prise en charge diagnostique et thérapeutique des patients souffrant de gliome. L'hétérogénéité intratumorale, laquelle commence tout juste à être explorée en profondeur, soulève de nouvelles et de nombreuses problématiques cliniques et ajoute un degré de complexité supplémentaire qu'il va falloir élucider pour optimiser la prise en charge médicale des patients. $\diamond$ Adult diffuse glioma: genetic heterogeneity and therapeutic implications

\section{LIENS D'INTÉRÊT}

Les auteurs déclarent n'avoir aucun lien d'intérêt concernant les données publiées dans cet article.

\section{RÉFÉRENCES}

1. Central brain tumor registry of the United States. www. cbtrus.org (consulté le 26 avril 2012).

2. Van den Bent MJ. Interobserver variation of the histopathological diagnosis in clinical trials on glioma : a clinician's perspective. Acta Neuropathol $2010 ; 120$ : 297-304.

3. Smith JS, Perry A, Borell TJ, et al. Alterations of chromosome arms $1 p$ and $19 q$ as predictors of survival in oligodendrogliomas, astrocytomas, and mixed oligoastrocytomas. J Clin Oncol 2000 ; 18 : 636-45.

4. Quon H, Abdulkarim B. Adjuvant treatment of anaplastic oligodendrogliomas and oligoastrocytomas. Cochrane Database Syst Rev 2008 : CD007104.

5. Houillier C, Wang X, Kaloshi G, et al. IDH1 or IDH2 mutations predict longer survival and response to temozolomide in low-grade gliomas. Neurology 2010 ; $75: 1560-6$.

6. Hegi ME, Diserens AC, Godard S, et al. Clinical trial substantiates the predictive value of 0-6methylguanine-DNA methyltransferase promoter methylation in glioblastoma patients treated with temozolomide. Clin Cancer Res 2004 ; 10 : 1871-4.

7. Everhard S, Kaloshi G, Criniere $\varepsilon$, et al. MGMT methylation: a marker of response to temozolomide in low-grade gliomas. Ann Neurol $2006 ; 60: 740$ - 3 .

8. Snuderl M, Fazlollahi L, Le LP, et al. Mosaic amplification of multiple receptor tyrosine kinase genes in glioblastoma. Cancer Cell $2011 ; 20: 810-7$.

9. Juillerat-Jeanneret L, Bernasconi CC, Bricod C, et al. Heterogeneity of human glioblastoma : glutathioneS-transferase and methylguanine-methyltransferase. Cancer Invest $2008 ; 26: 597-609$.
10. Bonavia R, Inda MM, Cavenee WK, et al. Heterogeneity maintenance in glioblastoma: a social network. Cancer Res $2011 ; 71: 4055-60$.

11. Yung WK, Vredenburgh JJ, Cloughesy TF, et al. Safety and efficacy of erlotinib in first-relapse glioblastoma: a phase II open-label study. Neuro Oncol 2010 ; 12 : 106170.

12. Paulsson J, Lindh MB, Jarvius M, et al. Prognostic but not predictive role of platelet-derived growth factor receptors in patients with recurrent glioblastoma. Int J Cancer $2010 ; 128$ : 1981-8.

13. Natsume A, Kinjo S, Yuki $K$, et al. Glioma-initiating cells and molecular pathology: implications for therapy. Brain Tumor Pathol $2011 ; 28$ : 1-12.

14. Ducray F, Mokhtari K, Criniere $\varepsilon$, et al. Diagnostic and prognostic value of alpha internexin expression in a series of 409 gliomas. Eur J Cancer $2011 ; 47: 802-8$.

15. SongTao $Q$, Lei $Y$, Si G, et al. IDH mutations predict longer survival and response to temozolomide in secondary glioblastoma. Cancer Sci 2011 ; 103 : 269-73.

16. Van den Bent MJ, Dubbink HJ, Sanson M, et al. MGMT promoter methylation is prognostic but not predictive for outcome to adjuvant PCV chemotherapy in anaplastic oligodendroglial tumors: a report from EORTC Brain Tumor Group Study 26951. J Clin Oncol 2009 ; 27 : 5881-6.

17. Jha P, Suri V, Jain A, et al. 06-methylguanine DNA methyltransferase gene promoter methylation status in gliomas and its correlation with other molecular alterations: first Indian report with review of challenges for use in customized treatment. Neurosurgery 2010 ; 67 : 1681-91.

18. Zawlik I, Vaccarella S, Kita D, et al. Promoter methylation and polymorphisms of the MGMT gene in glioblastomas: a population-based study. Neuroepidemiology $2009 ; 32: 21-9$.

19. Singh SK, Clarke ID, Terasaki M, et al. Identification of a cancer stem cell in human brain tumors. Cancer Res $2003 ; 63: 5821-8$.

20. Molina JR, Hayashi Y, Stephens C, et al. Invasive glioblastoma cells acquire stemness and increased Akt activation. Neoplasia $2010 ; 12:$ 453-63.

21. Hoelzinger DB, Mariani L, Weis J, et al. Gene expression profile of glioblastoma multiforme invasive phenotype points to new therapeutic targets. Neoplasia 2005 ; 7 : 7-16.

22. Gole B, Huszthy PC, Popovic M, et al. The regulation of cysteine cathepsins and cystatins in human gliomas. Int J Cancer 2012 (online). doi : 10.1002/ijc.27453.

23. Mariani L, Beaudry C, McDonough WS, et al. Deathassociated protein 3 (Dap-3) is overexpressed in invasive glioblastoma cells in vivo and in glioma cell lines with induced motility phenotype in vitro. Clin Cancer Res $2001 ; 7: 2480-9$.

24. Seidel S, Garvalov BK, Wirta V, et al. A hypoxic niche regulates glioblastoma stem cells through hypoxia inducible factor 2 alpha. Brain 2010 ; 133 : 983-95.

25. Rong $Y$, Durden DL, Van Meir EG, et al. Pseudopalisading necrosis in glioblastoma: a familiar morphologic feature that links vascular pathology, hypoxia, and angiogenesis. J Neuropathol Exp Neurol 2006 ; 65 : 529-39.

26. Charalambous C, Chen TC, Hofman FM. Characteristics of tumor-associated endothelial cells derived from glioblastoma multiforme. Neurosurg Focus $2006 ; 20$ : દ22.

27. Miebach S, Grau S, Hummel V, et al. Isolation and culture of microvascular endothelial cells from gliomas of different WHO grades. J NeuroOncol 2006 ; 76 : 39-48

28. Dahlstrand J, Collins VP, Lendahl U. Expression of the class VI intermediate filament nestin in human central nervous system tumors. Cancer Res 1992 ; 52 : 5334-41.

29. Shih $A H$, Holland $\varepsilon C$. Notch signaling enhances nestin expression in gliomas. Neoplasia $2006 ; 8$ : 1072-82. 